\title{
ESTADO NUTRICIONAL DE PACIENTES EN DOS CENTROS HOSPITALARIOS DE VILLAVICENCIO, COLOMBIA, 2015
}

\author{
Norton Pérez Gutiérrez ${ }^{1}$, Kellys Mileidys Ariza Rienzo² , Holmes Daniel Carvajal \\ Holguín ${ }^{3}$, Alejandro Jiménez Cardozo ${ }^{4}$, Jordi Piñeros Arias ${ }^{5}$, Diana Beltrán ${ }^{6}$, \\ Fabián Rodríguez López ${ }^{7}$, Angélica María Pérez ${ }^{8}$
}

\section{Resumen}

Objetivo. Establecer el estado nutricional en pacientes de dos instituciones hospitalarias de Villavicencio.

Método. Estudio de corte transversal, prospectivo, mediante una encuesta en 2 instituciones hospitalarias de Villavicencio, Colombia, en febrero de 2015. Se incluyeron pacientes adultos hospitalizados en servicios quirúrgicos. Los datos fueron registrados en Excel y se realizaron análisis estadísticos con SPSS a fin de encontrar asociaciones entre las variables de estudio y los desenlaces. El nivel de significancia se estableció cuando la p $<0,05$ mediante la prueba de Chi cuadrado y la t de Student.

Resultados. Se entrevistaron 116 pacientes. El 56\% de los pacientes fueron del hospital público, $58,8 \%$ fueron de servicios quirúrgicos y $65 \%$ fueron hombres. La edad promedio fue $65,7( \pm 20,6)$ años. Solo $37,1 \%$ de los pacientes tenía el peso registrado en la historia clínica, por lo cual solo se pudo determinar el estado nutricional al $31 \%$ de los encuestados. Solo se pudo determinar un 23,1\% de malnutrición, entre los cuales había un $9,5 \%$ de bajo peso. Los pacientes con mayor estancia hospitalaria fueron los del hospital público, en condición de desnutrición, sobrepeso y obesidad. No se pudo establecer asociación con mortalidad.

Conclusiones. La desnutrición es una enfermedad de alta prevalencia en pacientes hospitalizados pero no es diagnosticada frecuentemente. Las medidas antropométricas no son registradas en todas las historias clínicas de los pacientes hospitalizados y se requiere un mayor nivel de sensibilización de los trabajadores de salud para establecer medidas de intervención temprana a fin de obtener mejores desenlaces clínicos.

Palabras clave: estado nutricional, evaluación nutricional, encuestas nutricionales, necesidades nutricionales, desnutrición.

${ }^{1}$ Médico, Especialista en Cirugía, Subespecialista en medicina crítica y cuidado intensivo. Universidad Cooperativa de Colombia, docente facultad de medicina. Corporación Clínica Universidad Cooperativa de Colombia, especialista clínico Hospital Departamental de Villavicencio, coordinador unidad funcional de medicina crítica y cirugía. Correo Electrónico: norton.perez@hotmail.com

${ }^{2}$ Nutricionista y Dietista. Corporación Clínica Universidad Cooperativa de Colombia.

${ }^{3}$ Estudiante de medicina. Universidad Cooperativa de Colombia.

${ }^{4}$ Estudiante de medicina. Universidad Cooperativa de Colombia.

${ }^{5}$ Estudiante de medicina. Universidad Cooperativa de Colombia.

${ }^{6}$ Estudiante de medicina. Universidad Cooperativa de Colombia.

${ }^{7}$ Estudiante de medicina. Universidad Cooperativa de Colombia.

${ }^{8}$ Nutricionista y Dietista. Asociación Colombiana de Nutrición Clínica. 


\title{
NUTRITIONAL STATUS IN PATIENTS OF TWO HOSPITALS IN VILLAVICENCIO, COLOMBIA, 2015
}

\author{
Norton Pérez Gutiérrez, Kellys Mileidys Ariza Rienzo, Holmes Daniel Carvajal \\ Holguín, Alejandro Jiménez Cardozo, Jordi Piñeros Arias, Diana Beltrán, \\ Fabián Rodríguez López, Angélica María Pérez
}

\section{Abstract}

Objective. To determine the nutritional status and outcomes of patients in 2 hospitals in Villavicencio.

Method. Prospective cross-sectional study, survey conducted in two hospitals in Villavicencio, Colombia, on February 24th, 2015. Adult patients from surgical or clinical wards were included. Data was registered in Excel and statistical analysis with SPSS was conducted to determine association between independent variables and outcomes (Mortality and length of stay). Significance was established as $\mathrm{p}<0,05$ with Chi square and Student $\mathrm{T}$ test.

Results. One hundred and sixteen patients were surveyed. Fifty-six percent of patients were from the public hospital, 58,5\% were from surgical wards and $65 \%$ were men. Average age was $65,7( \pm 20,6)$ years old. Only $37,1 \%$ of the patients had the weight registered in the clinical chart, so nutritional status could be determined in only $31 \%$. Only $23,1 \%$ of malnutrition was found, including a $9,5 \%$ of underweighted patients. Longer length of stay was found in patients from the public hospital, as well as, under-nutrition, overweight and obesity. No association with mortality was found.

Conclusions. Under-nutrition is a prevalent disease in hospitalized patients, but it is not often diagnosed. Anthropometric measures are not registered in all clinical charts of hospitalized patients and it is required a higher level of sensitivity among health care workers, in order to establish early intervention measures, and to get better clinical outcomes. 


\title{
ESTADO NUTRICIONAL DE PACIENTES EM DOIS HOSPITAIS DE VILLAVICENCIO, COLÔMBIA, 2015
}

\author{
Norton Pérez Gutiérrez, Kellys Mileidys Ariza Rienzo, Holmes Daniel Carvajal \\ Holguín, Alejandro Jiménez Cardozo, Jordi Piñeros Arias, Diana Beltrán, \\ Fabián Rodríguez López, Angélica María Pérez
}

\section{Resumo}

Objetivo. Estabelecer o estado nutricional em pacientes de dos hospitais de Villavicencio.

Metodologia. Estudo de corte transversal, prospectivo, através de pesquisa em 2 hospitais de Villavicencio, Colômbia, em fevereiro de 2015. Pacientes adultos internados em serviços cirúrgicos foram incluídos. Os dados foram registrados em Excel y análise estadística com SPSS foi realizada a fim de encontrar associações entre as variáveis de estudo e os resultados. O nível de significância foi estabelecido quando $\mathrm{p}<0,05$ através do teste Qui Quadrado e a distribuição t de Student.

Resultados. 16 pacientes foram entrevistados. $56 \%$ dos pacientes eram do hospital público, $58,8 \%$ foram de serviços cirúrgicos e $65 \%$ eram homens. A idade média foi de $65,7(20,6 \pm)$ anos. Apenas $37,1 \%$ dos pacientes tiveram o peso registado na sua ficha, por isso só foi possível determinar o estado nutricional de $31 \%$ dos entrevistados. Foi possível determinar apenas 23,1\% de desnutrição, entre os quais havia $9,5 \%$ de baixo peso. Os pacientes com maior tempo de internação foram aqueles do hospital público, com desnutrição, sobrepeso e obesidade. Não foi possível estabelecer associação com a mortalidade.

Conclusões. A desnutrição é uma doença altamente prevalente em pacientes hospitalizados, mas muitas vezes não é diagnosticada. As medidas antropométricas não são registradas em todas as histórias clínicas de pacientes hospitalizados e um maior nível de sensibilização dos trabalhadores de saúde é necessário para estabelecer medidas de intervenção precoce para obter melhores resultados clínicos.

Palavras-chave: estado nutricional, avaliação nutricional, pesquisas nutricionais, necessidades nutricionais, desnutrição. 


\section{Introducción}

La desnutrición crónica y aguda afecta a pacientes en instituciones hospitalarias, como ha sido demostrado en países de Latinoamérica como Perú (1), Brasil (2,3), México (4), al igual que en algunos países desarrollados (5). No se conoce su prevalencia en la región y sin embargo tiene impacto en la morbilidad y la mortalidad. Estudios previos han demostrado que los pacientes con desnutrición tienen mayor riesgo de mortalidad y prolongada estancia hospitalaria (6-10). Adicionalmente, es poco reconocida, estudiada e intervenida por el personal de salud, debido a que la patología principal por la que el paciente consulta distrae y concentra toda la atención del clínico (11). En muchas ocasiones no se cuenta con suficiente recurso humano capacitado en temas de nutrición en las instituciones de la región. La encuesta realizada en el estudio Nutrition Day busca detectar los niveles de desnutrición hospitalaria y ha sido realizada previamente en América Latina, pero Colombia, y especialmente Villavicencio, hasta este año no ha participado $(2,3)$. No se conoce la prevalencia de malnutrición en hospitales de Colombia, ni mucho menos de la región.

La hipótesis manejada fue que la prevalencia de pacientes malnutridos es cercana al 50\%, y está asociada a peores desenlaces (mortalidad y estancia).

El objetivo del estudio fue establecer el estado nutricional en pacientes de insti-

1712 tuciones hospitalarias de Villavicencio y sus desenlaces.

\section{Materiales y método}

Se diseñó un estudio analítico, de corte transversal, prospectivo en 2 instituciones hospitalarias de alta complejidad de la ciudad de Villavicencio, Colombia, el día 24 de febrero de 2015. Para tal fin se encuestaron pacientes adultos, o en su defecto, menores de edad (adolescentes) cuyos tutores legales dieran el consentimiento para participar en la encuesta.

Los participantes fueron pacientes de servicios quirúrgicos y no quirúrgicos hospitalizados ese día en el Hospital Departamental de Villavicencio o en la Corporación Clínica Universidad Cooperativa de Colombia de la ciudad de Villavicencio, Colombia. Se excluyeron del estudio los pacientes que con orden de salida, las mujeres gestantes y los pacientes que no se pudieran incorporar para la medición de las medidas antropométricas.

Las encuestas fueron diligenciadas en formato pre-establecido mediante el diligenciamiento de una encuesta ya validada a nivel nacional y a nivel mundial en estudios previos del Nutrition Day $(2,3)$, y los resultados fueron exportados a una tabla de Excel formateada con las opciones de las variables en estudio.

La muestra fue seleccionada por método no probabilístico, no apareada de casos continuos por conveniencia, de acuerdo con los criterios de inclusión y exclusión. $\mathrm{El}$ análisis estadístico de las variables independientes y dependiente (estado nutricional, estancia, estado al egreso) se realizó mediante el programa estadístico SPSS versión 15 cuyo fin fue establecer las asociaciones entre los subgrupos. Se 
determinó como significancia la $\mathrm{p}<0,05$ mediante la prueba de Chi cuadrado y la t de Student para establecer la asociación entre las variables de estudio y los desenlaces (mortalidad y estancia). No se realizó análisis de regresión multivariado por los bajos números de pacientes en los subgrupos.

\section{Aspectos éticos}

Se trató de un estudio con riesgo mínimo para los participantes, en los cuales no se hizo ningún tipo de intervención y la participación se limitó a responder la encuesta que era diligenciada por uno de los investigadores. Luego de explicado el objetivo del estudio, las personas podían manifestar su deseo de participar o no y se dio por entendido su deseo, al diligenciar la encuesta. No se cuantificaron los desistimientos. El estudio fue aprobado por el comité de investigaciones de las instituciones participantes, de la facultad de medicina de la Universidad Cooperativa de Colombia y de la Asociación Colombiana de Nutrición Clínica. Las instituciones involucradas dieron su aprobación para participar, incluso por medio del apoyo de algunos de sus profesionales quienes se incorporaron como investigadores. Los resultados del estudio fueron dados a conocer a las instituciones en reunión cerrada e independiente.

\section{Resultados}

Se entrevistaron 116 pacientes (Ver Tabla 1). El 48,3\% de los participantes fueron del hospital B, 58,6\% fueron de servicios quirúrgicos y $56 \%$ fueron hombres. La edad promedio fue $65,7 \pm$ 20,6 años y el $45,7 \%$ fueron mayores de 65 años (Ver Figura 1).

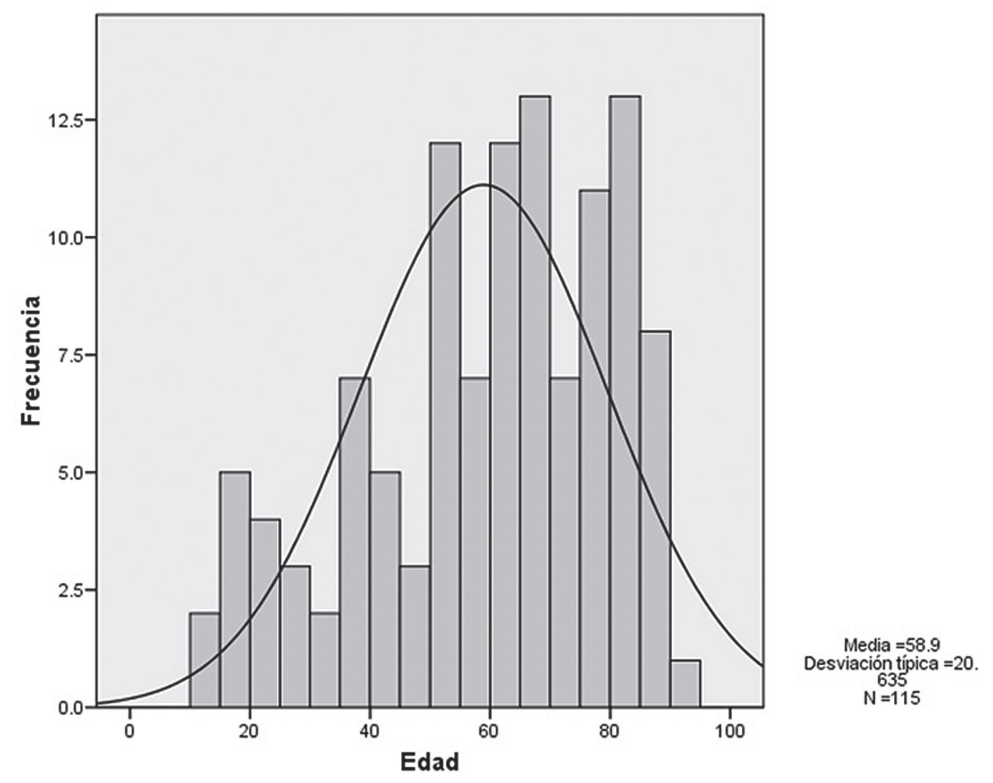

Figura 1. Distribución de la edad en pacientes encuestados durante el Nutrition Day en Villavicencio, 
INVESTIGACIONES ANDINA No. 33 Vol. 18

Tabla 1. Características de los pacientes encuestados durante el Nutrition Day en Villavicencio, Colombia, 2015. DE = Desviación estándar. IMC = índice de masa corporal.

\begin{tabular}{l|c|c|c|c|c|c}
\hline \multicolumn{1}{c|}{ Variable } & N & $\%$ & Promedio & DE & Mínimo & Máximo \\
\hline Institución & 116 & 100,0 & & & \\
A & 60 & 51,7 & & \\
B & 56 & 48,3 &
\end{tabular}

\section{Servicio}

$\begin{array}{lll}\text { Médico } & 48 & 41,4 \\ \text { Quirúrgico } & 68 & 58,6\end{array}$

Sexo

$\begin{array}{lrr}\text { Hombre } & 65 & 56,0 \\ \text { Mujer } & 51 & 44,0\end{array}$

Edad

$65,7 \quad 20,6 \quad 12 \quad 91$

$\begin{array}{lcccccc}\text { Adultos mayores (>=65 años) } & 53 & 45,7 & & & \\ \text { Peso } & 43 & 37,1 & 65,6 & 15,4 & 38 & 95 \\ \text { Talla } & 36 & 31,0 & 161,4 & 7,7 & 150 & 180 \\ \text { IMC } & 36 & 31,0 & 25,4 & 5,5 & 16 & 40 \\ \text { Tiempo hospitalización previo } & 115 & 99,1 & 13,3 & 23,0 & 1 & 180 \\ \text { Estado nutricional } & 37 & 31.9 & & & \\ \quad \text { Bajo peso } & 11 & 9.5 & & & \\ \quad \text { Normal } & 9 & 7.8 & & & \\ \quad \text { Sobrepeso } & 4 & 3.4 & & & \\ \quad \text { Obesidad } & 13 & 11.2 & \end{array}$

Solo el $37,1 \%$ de los pacientes tenía registro del peso en la historia clínica y $31 \%$ de la talla. No se tomaron medidas antropométricas durante la encuesta. El peso promedio fue 65,6 $\pm 15,4$ kilos, la talla $161,4 \pm 7,7$ y el índice de masa corporal (IMC) 25,4 $\pm 5,5$.

El tiempo de hospitalización previo a la encuesta fue de 13,3 $\pm 23,0$ días con un rango entre 1 y 180 días y el $14,7 \%$ de los 
pacientes habían estado en UCI. El mismo porcentaje de pacientes estaban a la espera de una cirugía y el 31,9\% habían sido ya intervenido hacía $14,9 \pm 26,1$ días en promedio.

El 50,9\% de los encuestados hacía recordación del peso hace 5 años y manifestaron que tenían un promedio de 72,6 $\pm 14,3$ kilos en esa época. El 62,1\% refirieron pérdida de peso en los últimos 3 meses, pero solo el 58,6\% pudo estimar la magnitud que estuvo en 7,6 $\pm 4,6 \mathrm{ki}$ los en promedio. El 25\% de los pacientes tomaba 5 o más fármacos.

Al preguntar sobre la ingesta durante la última semana dentro o fuera del hospital, solo $35,3 \%$ manifestaron que fue normal, mientras que $41,3 \%$ fue la mitad o menos y la principal causa fue falta de apetito (34\%), seguida de otras, entre las que se destacó el sabor de los alimentos (22\%). Al evaluar visualmente la ingesta del almuerzo o la cena el día de la entrevista se encontró que solo el $31 \%$ se comió la ración completa, no obstante, el 47,4\% de los encuestados manifestó que su apetito era normal ese día. Para ello se debe tener en cuenta que el 25,9\% de los encuestados no podía deambular y otro $23,3 \%$ necesitaba ayuda. El 19\% percibe que su salud es mala y el $47,4 \%$ que es regular.

La procedencia solo se pudo establecer en el $41,4 \%$ de los pacientes, de los cuales el 75\% eran de Villavicencio, mientras que el 10,5\% manifestó provenir de otros departamentos (Ver Figura 2). Solo $4,3 \%$ de los pacientes pertenecían al régimen contributivo y en total estaban cubiertos por 9 aseguradoras.

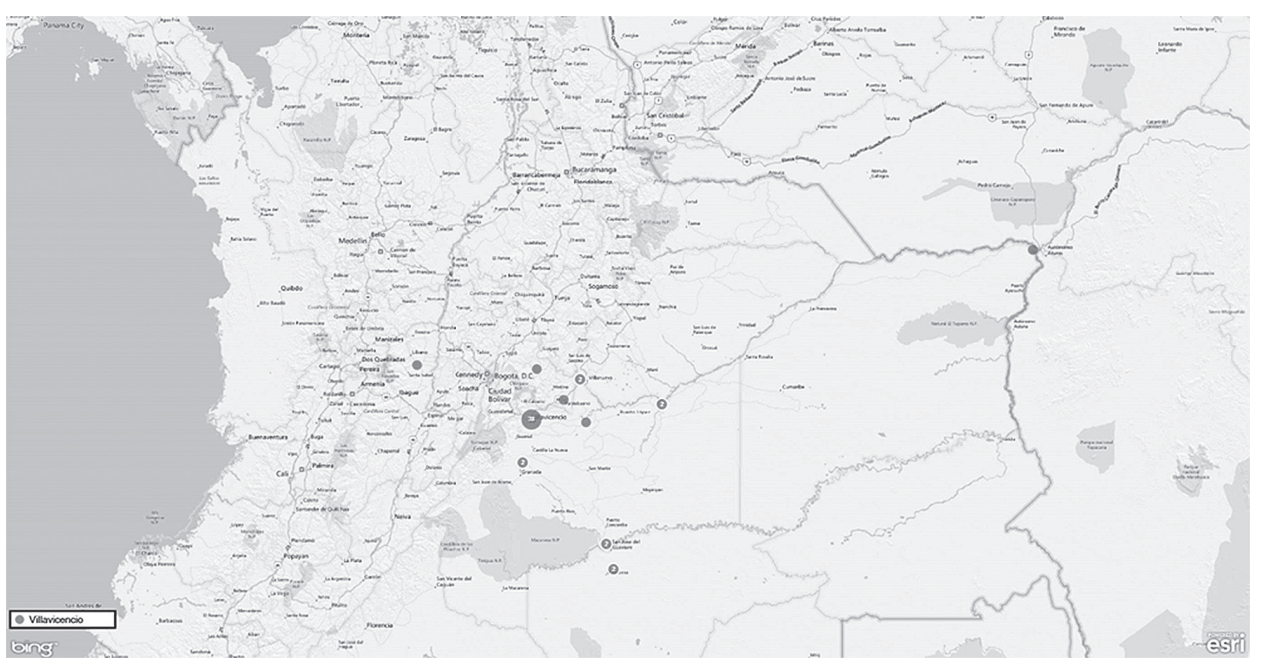

Figura 2. Mapa de geo-referenciación de la procedencia de pacientes encuestados durante el Nutrition Day en Villavicencio, Colombia, 2015.

Los órganos o sistemas afectados por las patologías se describen en la Tabla 2. 
INVESTIGACIONES ANDINA No. 33 Vol. 18

Tabla 2. Tipo de patologías en pacientes hospitalizados durante el Nutrition Day 2014 en Villavicencio.

\begin{tabular}{l|c|c}
\hline \multicolumn{1}{c|}{ Órganos Afectados } & $\mathbf{N}$ & \% \\
\hline Neurológico & 15 & 12.9 \\
Nariz y garganta & 2 & 1.7 \\
Cardiocirculatorio & 20 & 17.2 \\
Respiratorio & 18 & 15.5 \\
Hígado & 4 & 3.4 \\
Gastrointestinal & 17 & 14.7 \\
Urinario & 12 & 10.3 \\
Endocrino & 10 & 8.6 \\
Osteomuscular & 22 & 19.0 \\
Hematológico & 3 & 2.6 \\
Piel & 10 & 8.6 \\
Isquemia & 1 & 0.9 \\
Cáncer & 2 & 1.7 \\
Infeccioso & 6 & 5.2 \\
\hline
\end{tabular}

Se pudo determinar el estado nutricional en 37 pacientes, de los cuales el 23,1\% se encontraban en estado de malnutrición y el 9,5\% registraron bajo peso. La estancia hospitalaria fue 23,1 $\pm 31,0$ días en promedio y al hacer el seguimiento se encontró que $6 \%$ habían fallecido al egreso (Ver Tabla 3).

Tabla 2. Desenlaces de los pacientes encuestados durante el Nutrition Day en Villavicencio, 2015. $\mathrm{DE}=$ Desviación estándar.

\begin{tabular}{l|c|c|c|c|c|c}
\hline \multicolumn{1}{c|}{ Variable } & N & $\%$ & Promedio & DE & Mínimo & Máximo \\
\hline Estancia & 108 & & 23,1 & 31,0 & 2 & 268 \\
Estado al egreso & & & & & \\
$\quad$ Vivo & 109 & 94,0 & & \\
$\quad$ Muerto & 7 & 6,0 & & \\
\hline
\end{tabular}


El peso y la talla también fueron mayores en hombres que en mujeres y por consiguiente en el IMC aunque la diferencia no fue significativa $(25,5 \pm 4,9$ vs $25,3 \pm$ $6,4)$. El peso de 5 años atrás fue también mayor en hombres que en mujeres y en el 51,4 vs $48,6 \%$ se encontró pérdida de peso en los últimos 3 meses.

El tiempo promedio de hospitalización previa fue más largo en hombres que en mujeres $(15,7 \pm 29,4$ vs 10,2 \pm 9,8 días $)$. Del mismo modo, quienes estuvieron internados en la UCI fueron mayormente hombres ( 58,8 vs $41,2 \%$ ), sin embargo, la población en espera de una cirugía fue mayormente femenina $(29,4$ vs $70,6 \%)$. No hubo diferencia entre la proporción, por género, de aquellos intervenidos quirúrgicamente, pero el tiempo post-operatorio fue mucho mayor en hombres que en mujeres (21,2 \pm 34 vs $8,3 \pm 11$ días).

Mientras las mujeres tuvieron mayor proporción de bajo peso $(54,5 \%)$ la obesidad fue más frecuente en hombres $(53,8 \%)$, pero no hubo diferencias significativas $(p>0,05)$. La mortalidad fue mayor en hombres que en mujeres (4,3 vs $1,7 \%)$, sin embargo no hubo significancia estadística $(p=0,33)$. Tampoco hubo diferencias en la estancia por género.

De igual forma, la edad promedio fue mayor en $A(65,7 \pm 17,9$ vs $51,7 \pm 21,1)$. La proporción de pacientes mayores de 65 años fue mayor en A (55,9 vs $35,7 \%)$. La proporción de pacientes no quirúrgicos fue mayor en A $(66,7 \%)$, mientras que la de quirúrgicos fue mayor en $\mathrm{B}(58,8 \%)$. El peso promedio fue mayor en los pacientes de A (70,5 $\pm 13,1$ vs $59,3 \pm 16,1$ kilos), aunque la talla fue menor $(161,1 \pm 6,1$ vs $161,7 \pm$ 9,4 centímetros). Los pacientes tuvieron mayor estancia hospitalaria en $\mathrm{B}$ (Ver Figura 3), pero fue menor en los servicios no quirúrgicos $(p=0,2)$, (Ver Figura 4).

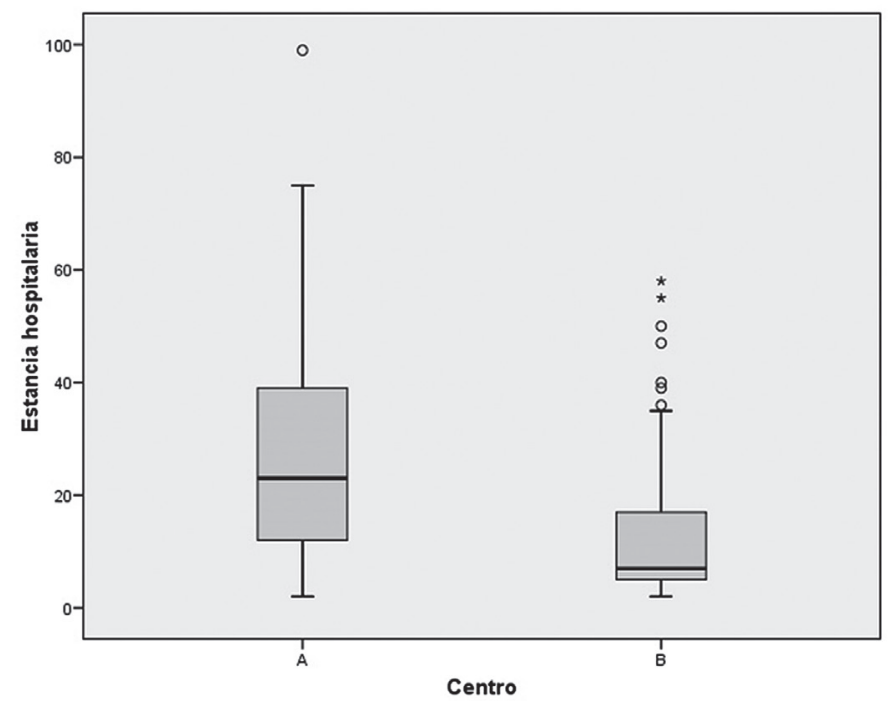

Figura 3. Comparación de la estancia entre las instituciones participantes en la encuesta del Nutrition 


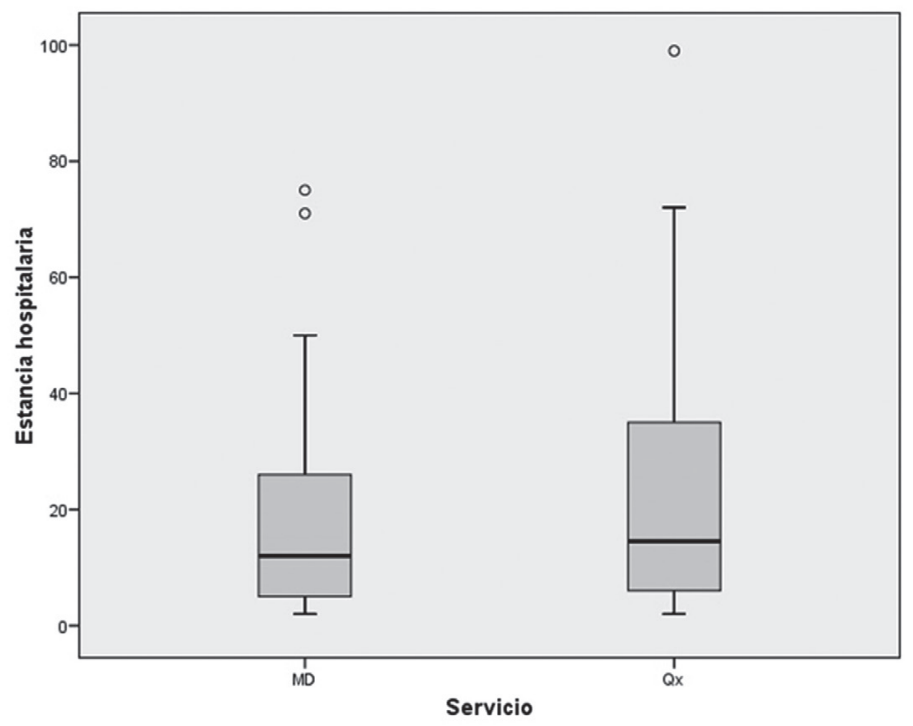

Figura 4. Comparación de la estancia entre tipos de servicios durante la encuesta Nutrition Day en Villavicencio, Colombia, 2015 ( $p=0,2$; prueba U de Mann-Whitney).

El número de pacientes que estuvieron previamente en UCI fue mayor en el hospital B (64,7\%), mientras que hubo un mayor número de pacientes en espera de cirugía en el hospital A (70,6 vs $29,4 \%)$. El promedio de días post-operatorios fue mayor en el hospital B 11,9 $\pm 10,5$ vs $16,7 \pm 32,9$. El peso recordado fue mayor en los pacientes de A (74,5 \pm $12,5$ vs $89,3 \pm 16,7)$ y un mayor número de pacientes de A reportó pérdida de peso en los últimos 3 meses (62,5 vs $37,5 \%)$, sin embargo la pérdida fue mayor en $B(6,9 \pm 3,7$ vs $9,1 \pm 4,9)$.

La ingesta fue baja en ambas instituciones pero fue mayor en $A$, ya que solo fue normal en 41,5 vs $58,5 \%$ respectivamente. En el día de la encuesta, se evidenció que solo el 50\% tuvo una ingesta normal en ambas instituciones. Hubo una menor proporción de pacientes con peso bajo en A (33,3 vs $66,7 \%)$.

\section{Desenlaces}

La estancia fue mucho mayor en B (14 $\pm 14,5$ vs $31,8 \pm 39,2 ; p<0,001)$. También fue mayor en los pacientes que estuvieron en UCI $(p=0,0009)$, (Ver Figura 5). 


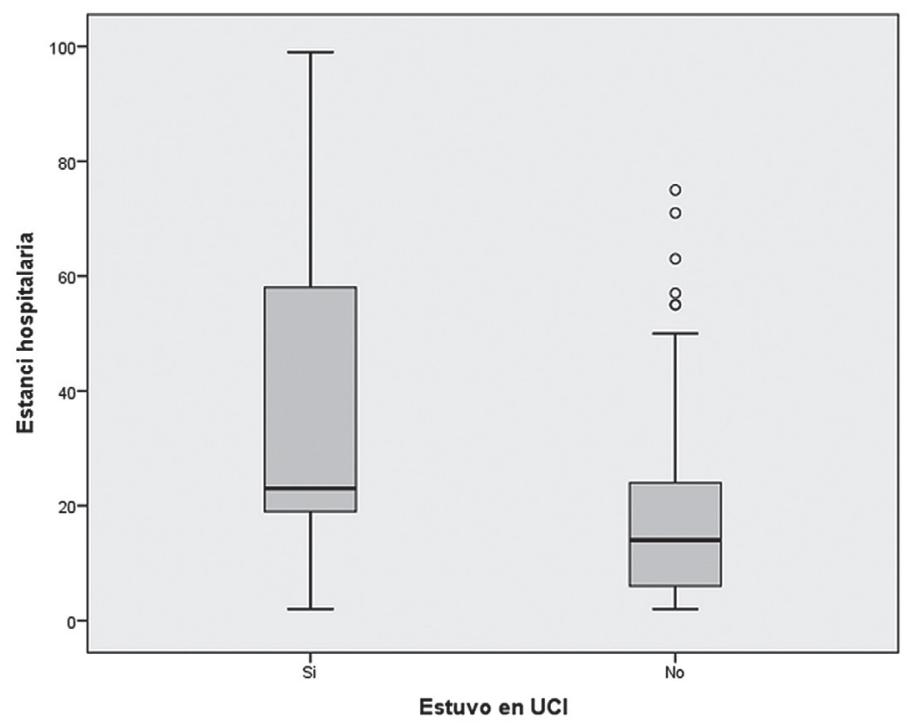

Figura 5. Estancia hospitalaria de pacientes que estuvieron en UCI $(p=0,0009)$.

Por otro lado, los pacientes con desnutrición, sobrepeso y obesidad tuvieron mayor promedio de estancia hospitalaria que los pacientes con peso normal (Ver Figura 6), aunque no se pudieron detectar diferencias significativas $(\mathrm{p}=0,481)$, proba- blemente por efecto de los bajos números en los sub grupos. Tampoco se pudo demostrar mayor aumento de la mortalidad en los pacientes malnutridos $(\mathrm{p}=0,205) \mathrm{ni}$ de aquellos con pérdida de peso durante los últimos 3 meses $(\mathrm{p}=230)$.

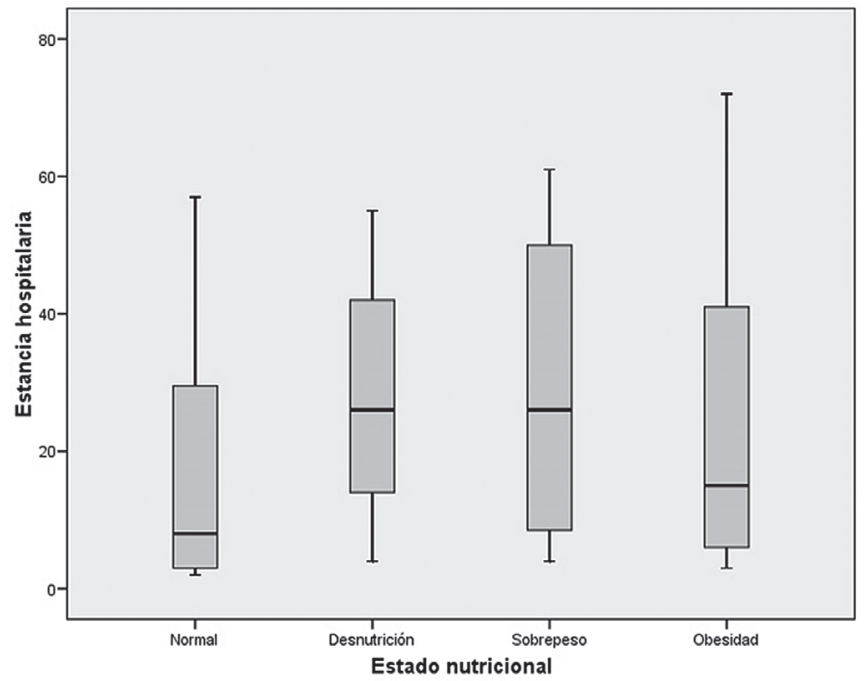

Figura 6. Comparación de la estancia hospitalaria según el estado nutricional en pacientes entrevistados en el Nutrition Day en Villavicencio, Colombia, 2015 ( $p=0$,2; prueba de Kruskal-Wallis). 
En el análisis de regresión lineal se observó una correlación de los días de estancia con el tiempo de hospitalización previo a la encuesta $(p<0,001)$, pero no con el número de días de cirugía $(\mathrm{p}=0,37)$.

\section{Discusión}

La desnutrición tiene una prevalencia importante desde la niñez en Colombia y está asociada a factores socio-económicos (12-21). En la edad avanzada esta situación nuevamente adquiere importancia, debido a la asociación con enfermedades crónicas y debilitantes $(22,23)$. El cuidado en hogares alternos se asocia con mayores tasas de desnutrición y morbilidad de modo tal, que la desnutrición hospitalaria no es, en muchas oportunidades, un problema nosocomial sino previo (24). Un estudio realizado en Ginebra y Berlín encontró una prevalencia alta de malnutrición, al momento del ingreso hospitalario, comparado con voluntarios sanos de la misma edad y talla y adicionalmente que el índice de masa corporal (IMC) y la albumina sérica sub-valoraba el estado de malnutrición (11). Por lo anterior, se debe considerar que la comida hospitalaria no es un problema de hotelería, sino de gestión terapéutica (25).

Estos factores se acumulan en los pacientes hospitalizados, además de otros propios de la atención y la enfermedad, lo cual empeora el estado previo y tiene un impacto determinante en la evolución, el desenlace clínico y los costos (26-28). En países que van mejorando sus niveles de desarrollo, el aumento del promedio de vida se ha encontrado con un aumento de la prevalencia de malnutrición como patología geriátrica $(20,27)$. En el estudio se encontró que el $53,4 \%$ de los pacientes tuvieron más de 65 años y en los casos en que se logró encontrar las mediciones para el cálculo del IMC se encontró en un promedio de $26,1( \pm 4,5)$ y una frecuencia de desnutrición del $7 \%$ en este grupo de edad.

La evaluación antropométrica se correlaciona con el peso y la talla en ancianos $(22,29)$. El IMC es un buen indicador del estado nutricional según la edad y el género $(22,30,31)$. Se considera que un IMC $<24$ es indicador de desnutrición y $>27$ de obesidad. En este caso, se debe tener en cuenta la medición de la talla, ya que la cifosis puede ser un factor de confusión, mientras que algunos autores han preferido la predicción mediante la envergadura de los brazos (32). Un 7\% adicional de pacientes mayores de 65 años tuvieron un IMC $>27$ en el estudio.

Aun así, son pocos los estudios de medición de este problema que se han hecho en nuestro medio (33). No se encontró ningún artículo de este tipo para la Orinoquia Colombiana. Si bien se han hecho estudios internacionales sobre este tema, incluida América Latina, Colombia no participó inicialmente $(2,3,34)$. El Nutrition Day es una iniciativa internacional que busca establecer el nivel de desnutrición de los pacientes hospitalizados y su impacto en los desenlaces. Ha sido realizado en varios países alrededor del mundo, incluyendo América Latina, pero Colombia solo participó hasta el año 2010 (2,3). En ninguno de ellos ha participado ninguna institución hospitalaria de Villavicencio. La importancia 
de este estudio, y el posible impacto que podría tener al divulgar los resultados al interior de las instituciones participantes, radica en haber encontrado que la frecuencia de medición de las variables antropométricas fue muy baja (31\%), a pesar de la valiosa información aportada, desde el ingreso hospitalario. En poblaciones con alto riesgo de malnutrición, como los adultos mayores, la frecuencia de medición fue solo del $29 \%$.

En el año 2010 se realizó el primer $\mathrm{Nu}$ trition Day en Colombia, organizado por la Universidad el Bosque, donde participaron 7 hospitales de Bogotá. En este estudio se encontró también que el paciente es pesado al ingreso únicamente en el $28 \%$ de los casos, a pesar de que el $58 \%$ de ellos manifestaron haber perdido peso durante los últimos 3 meses $(35,36)$. Al siguiente año se reclutaron 22 instituciones en 8 ciudades y participaron 1491 pacientes, de los cuales $61,6 \%$ informaron pérdida de peso en los últimos 3 meses y una mortalidad del 6,8\% a los 30 días (37). En el año 2012 participaron 25 instituciones de 7 ciudades y 1576 pacientes. En esta oportunidad el $51,9 \%$ manifestaron haber perdido peso en los últimos 3 meses y la mortalidad a los 30 días fue de 3,68\%, siendo el doble para los pacientes no quirúrgicos (38). En el año 2013 se encuestaron 1069 pacientes de los cuales el 54,1\% manifestaron pérdida de peso y se encontró una estancia promedio de 12 (2-128) días y mortalidad del 3,83\% (39). Ninguno de estos trabajos fue publicado en una revista científica para difusión de los resultados y solo uno de ellos (2010) fue objeto de una tesis de grado. En el presente estudio los pacientes con bajo peso tuvieron mayor tiempo de estancia hospitalaria en contraste con los que no lo estuvieron ( 47 vs 18 días), ( $\mathrm{p}=0,0003)$. En general los pacientes con malnutrición tuvieron mayor estancia hospitalaria (35 \pm 50 vs $18 \pm 19$ días), ( $\mathrm{p}=0,0039)$.

La desnutrición está asociada con la mayor mortalidad en niños enfermos, pacientes quirúrgicos y no quirúrgicos (40). Igualmente, se ha descrito mayor estancia hospitalaria en pacientes ortopédicos con desnutrición (41). La intervención nutricional ha demostrado modificar estos desenlaces. Algunas conductas, que son reevaluadas hoy en día, como el ayuno peri-operatorio prolongado, empeoran el estado previo del paciente (42). Actualmente, existe suficiente evidencia en la literatura, desde hace ya 2 décadas, que recomienda, al igual que los programas de calidad peri-operatoria, evitar el ayuno prolongado preoperatorio e iniciar la nutrición temprana postoperatoria (43-47). En este estudio no se logró demostrar una asociación del estado nutricional con la mortalidad, probablemente por la escasa población entre los subgrupos.

Varios estudios han demostrado el impacto de la nutrición en los desenlaces clínicos en diferentes tipos de pacientes como trauma, ortopedia, EPOC, medicina interna, cáncer, etc. por no mencionar los pacientes más críticos (48-59). Entre ellos, se ha demostrado el aumento de la estancia, las complicaciones, la mortalidad y los costos de la atención (60). Otro estudio similar demostró que los pacientes que ingieren menos de la cuarta parte de la alimentación hospitalaria tienen mayor riesgo de mortalidad 
(61). En el presente estudio se encontró que ninguno de los pacientes que comieron más de la mitad del alimento el día de la encuesta, falleció $(\mathrm{p}<0,001)$.

En el presente estudio se encontró una pérdida de peso en los últimos 3 meses de $62,1 \%$, un promedio de estancia de $23,1( \pm 31,0)$ días y mortalidad del 6\%. La estancia prolongada contrasta con la encontrada en los estudios previos en Colombia, que si bien el promedio es mayor que en el resto del mundo, también se encontraron valores atípicos de estancias máximas (Mundial 12 [1-358];
Colombia 12 [2-128]; Villavicencio 23,1 [2-268]), (39). No se pudo demostrar otras asociaciones esperadas entre los pacientes malnutridos o con pérdida de peso en los últimos 3 meses y la estancia (Ver Figura 7), así como la mortalidad ( $\mathrm{p}=0,183$ ). Para ello se requiere mejorar la muestra en cada uno de los subgrupos en futuros estudios. El estudio tiene limitaciones por su diseño, la falta de muestreo y estandarización en las mediciones antropométricas, pero su enfoque no intervencionista refleja la realidad que se observa en el escenario clínico.

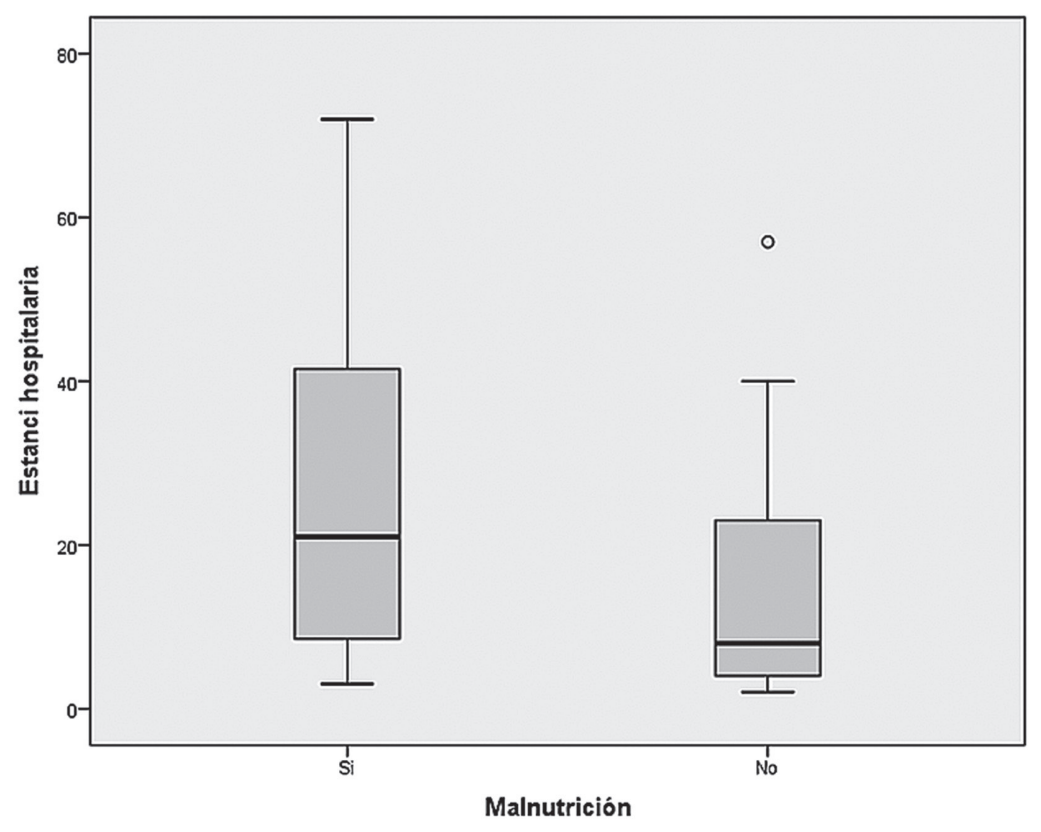

Figura 7. Estancia hospitalaria en pacientes con malnutrición encuestados en el Nutrition Day 2015 $(\mathrm{p}=0,188)$. 


\section{Conclusiones}

Colombia es un país en vía de desarrollo, con altos niveles de desnutrición en la población desde la infancia. Los pacientes hospitalizados, principalmente ancianos y con enfermedades crónicas y debilitantes, tienen mayor riesgo de aumentar el déficit nutricional $(12,62)$. Esta patología de alta prevalencia es poco percibida en el personal de salud lo cual disminuye el grado de alerta y las posibilidades de intervención temprana (63). Algunos autores muestran estrategias automatizadas en el registro de la historia clínica para detectar estos pacientes mediante los resultados de las mediciones $(64,65)$.

El estudio demuestra, en primer lugar, un bajo nivel de medición de variables antropométricas que son de importancia clínica para el manejo diario y monitorización, así como para el establecimiento del estado nutricional previo y la variación durante la hospitalización. Esta es una gran limitación esperada en el estudio, sin embargo, en aquellos casos en que se encontraron las variables registradas, se encontró una alta prevalencia de pacientes en estado de malnutrición $(24,1 \%)$ que está por debajo de mediciones previas en América Latina, lo cual hace pensar que se debe al sub registro $(26,66,67)$.

No se conocen aún los datos de la gran encuesta nacional realizada durante el Nutrition Day 2015, los cuales podrán ser contrastados con estos. Es posible que haya diferencias sustanciales debido a las características de la región y de las instituciones participantes, especial- mente en el hospital, que por su naturaleza pública atiende a la población más vulnerable.

Otra limitación del estudio se encuentra en el tipo de reclutamiento usado para la encuesta que incluyó algunos de los criterios de exclusión, los cuales pueden llevar a mayor sub registro y error tipo II por sesgo de selección. Adicionalmente se requiere una mayor homogeneidad entre los sub grupos para las comparaciones estadísticas.

A pesar de lo anterior, el estudio muestra de forma exploratoria que el problema de la malnutrición también está presente en nuestro medio y en una magnitud no bien establecida todavía (68). El ejercicio de esta primera medición permite plantear algunas hipótesis que deberán ser confirmadas en futuras mediciones con un mejor diseño, entre ellas: ¿Cuáles son los grupos de pacientes con mayor riesgo de desnutrición? Indígenas, adultos mayores de 65 años, según el tipo de aseguramiento. Un primer hallazgo en este trabajo es que los pacientes de patología no quirúrgica tienen mayor riesgo de malnutrición. Pero, ¿Hay diferencias entre los pacientes de ortopedia en contraste con los de cirugía general? (69).

En cualquier caso, se requiere mejorar los registros de las historias clínicas para incluir la medición de las variables antropométricas que son de importancia para el diagnóstico nutricional, su intervención temprana y una concientización del personal de salud en todas las especialidades clínicas y quirúrgicas de esta patología prevalente, que impacta los desenlaces clínicos pero cuya intervención temprana puede mitigar los efectos $(70,71)$. 


\section{Referencias}

1. Veramendi-Espinoza LE, Zafra-Tanaka $\mathrm{JH}$, Salazar-Saavedra O, Basilio-Flores JE, Millones-Sánchez E, Pérez-Casquino GA, et al. Prevalencia y factores asociados a desnutrición hospitalaria en un hospital general; Perú, 2012. Nutr Hosp. 2013;28(4):1236-43.

2. Waitzberg DL, Ravacci GR, Raslan M. Desnutrición hospitalaria. Nutr Hosp. 2011;26(2):254-64.

3. Correia MITD, Campos ACL. Prevalence of hospital malnutrition in Latin America: The multicenter ELAN study. Nutrition. 2003 Oct;19(10):823-5.

4. Baccaro F, Sánchez A. Determination of hospital malnutrition: a comparison between the subjective global assessment and body mass index. Rev Gastroenterol México. 2009;74(2):105-9.

5. Hiesmayr M, Frantal S, Schindler K, Themessl-Huber $M$, Mouhieddine $M$, Schuh $\mathrm{C}$, et al. The Patient- And Nutrition-Derived Outcome Risk Assessment Score (PANDORA): Development of a Simple Predictive Risk Score for 30-Day In-Hospital Mortality Based on Demographics, Clinical Observation, and Nutrition. PLoS One. 2015;10(5):e0127316.

6. Agarwal $E$, Ferguson $M$, Banks $M$, Batterham M, Bauer J, Capra S, et al. Malnutrition and poor food intake are associated with prolonged hospital stay, frequent readmissions, and greater in-hospital mortality: Results from the Nutrition Care Day Survey 2010. Clin Nutr. 2013;32(5):737-45.

7. Baccaro F, A S. Body mass index is a poor predictor of malnutrition in hospitalized patients. Niger J Med. 2015;24(4):310-4.

8. Álvarez-Hernández J, Planas Vila M, León-Sanz M, García de Lorenzo A, Celaya-Pérez S, García-Lorda P, et al. Preva lence and costs of malnutrition in hospitalized patients; the PREDyCES Study. Nutr Hosp. 2012;27(4):1049-59.

9. Sharma Y, Miller M, Shahi R, Hakendorf P, Horwood C, Thompson C. Malnutrition screening in acutely unwell elderly inpatients. Br J Nurs. 2016;25(18).

10. Wie GA, Cho YA, Kim SY, Kim SM, Bae JM, Joung $\mathrm{H}$. Prevalence and risk factors of malnutrition among cancer patients according to tumor location and stage in the National Cancer Center in Korea. Nutrition. 2010;26(3):263-8.

11. Kyle UG, Pirlich M, Schuetz T, Luebke HJ, Lochs H, Pichard C. Prevalence of malnutrition in 1760 patients at hospital admission: a controlled population study of body composition. Clin Nutr. 2003;22(5):473-81.

12. Argüello R, Cáceres M, Figueredo R. Desnutrición hospitalaria. Tendencias en Med. 2009;23-7.

13. Arias Ortiz NE. Desnutrición y bioética: reflexiones sobre un problema de salud pública. Rev Latinoam Bioet. 2012;12(1):28-35.

14. Ardila VA, Prada GE, Herrán ÓF. Distribución de energía y macronutrientes en hogares colombianos. Biomédica. 2013;33:163-74.

15. Cardona Arango D, Segura-Cardona A, Espinosa López A m. Mortalidad de adultos mayores por deficiencias nutricionales en los departamentos de Colombia. Rev Salud Pública. 2012;14(4):584-95.

16. Manrique Corredor EJ. Pobreza, desnutrición y reformas de salud en Colombia. Rev Salud Hist Sanid. 2011;6(1):1-14.

17. Manrique E, Salazar J. Dimensión social de la desnutrición, apuntes para la discusión. Cult Científica. 2012;10:84-9. 
18. Jiménez-Benítez D, Rodríguez-Martín A, Jiménez-Rodríguez R. Análisis de determinantes sociales de la desnutrición en Latinoamérica. Nutr Hosp. 2010;25(Supl $3): 18-25$.

19. Kac G, García Alvear JL, Red de Malnutrición en Iberoamérica del Programa de Clencia y Tecnología para el Desarrollo (Red Mel-CYTED). Epidemiología de la desnutrición en Latinoamérica: situación actual. Nutr Hosp. 2010;55(21):50-6.

20. Bernal Orozco MF Vizmanos B CDLR a J. La nutricion del anciano como un problema de salud publica. Antropo. 2008;16:43-55.

21. Viloria de la Hoz J. Nutrición en el Caribe Colombiano y su relación con el capital humano. 93rd ed. [Documentos de trabajo sobre economía regional] Bogotá: Banco de la República: Centro de Estudios Económicos Regionales (CEER). 2007; 66 p.

22. Becerra Bulla F. Tendencias actuales en la valoración antropométrica del anciano. Rev Fac Med Univ Nac Colomb. 2006;54(4):283-90.

23. Camina Martín M a., Barrera Ortega $\mathrm{S}$, Domínguez Rodríguez $\mathrm{L}$, Couceiro Muiño C, de Mateo Silleras B, Redondo del Río MP. Presencia de malnutrición y riesgo de malnutrición en ancianos institucionalizados con demencia en función del tipo y estadío evolutivo. Nutr Hosp. 2012;27(2):434-40.

24. Arvanitakis M, Beck A, Coppens P, De Man F, Elia M, Hebuterne $X$, et al. Nutrition in care homes and home care: How to implement adequate strategies [report of the Brussels Forum 22-23 November 2007]. Clin Nutr. 2008;27:481-8.

25. Elia M. Nutrition, hospital food and in-hospital mortality. Clin Nutr. 2009;28:481-3.

26. Arana Montoya IP, García WR. Estado nutricional de pacientes hospitalizados en medicina interna en un hospital de referencia. Reper Med Cir. 2011;20(1):24-9.

27. Gómez Ramos MJ, González Valverde FM. Alta prevalencia de la desnutrición en ancianos españoles ingresados en un hospital general y factores asociados. ALAN. 2005;55(1):71-6.

28. Culebras JM. Malnutrition in the twenty-first century: an epidemic affecting surgical outcome. Surg Infect (Larchmt). 2013 Jun;14(3):237-43.

29. Borba de Amorim $R$, Coelho Santa Cruz MA, Borges de Souza-Júnior PR, Corrêa da Mota J, González H C. Medidas de estimación de la estatura aplicadas al índice de masa corporal (IMC) en la evaluación del estado nutricional de adultos mayores. Rev Chil Nutr. 2008;35(Supl 1):272-9.

30. Puche RC. El índice de masa corporal y los razonamientos de un astrónomo. Buenos Aires. Medicina. 2005;65:361-5.

31. Berral de la Rosa FJ, del Aguila Quirós D. Valoración antropométrica/ nutricional de enfermos adultos hospitalizados o encamados. Arch Med Deport. 2002; XIX(88):129-35.

32. Salva Casanovas A, Serra Rexach JA, Cuesta Triana F, Planas Vila M, Wanden-Berghe C, Garcia Peris P, et al. Valoración nutricional en el anciano: Recomendaciones prácticas de los expertos en geriatría y nutrición. 2011.

33. Barrera $M$ del $P$, Pinilla AE, Caicedo LM, Castillo YM, Lozano YM, Rodríguez KM. Factores de riesgos alimentarios y nutricionales en adultos con diabetes mellitus. Rev Fac Med. 2012;60(Supl):28-40.

34. Matos AA, Sinclair J, García Mayorca E, Ochoa J. Prevalencia de la desnutrición hospitalaria en Panamá: Estudio Latinoamericano de Nutrición (ELAN). Rev Med 
35. Carvajal CM. Nutrition Day Colombia - Bogotá: Metodología y experiencia. Bogotá, D.C.; 2011. 77 páginas.

36. Quintero Gonzalez SP. Organización y ejecución del día de la nutrición en instituciones hospitalarias de Bogota, Colombia. Primera fase. Pontificia Universidad Javeriana; 2010.

37. Contreras CP. Resumen ejecutivo Nutrition Day Colombia 2011. Bogotá, D.C.; 2011.

38. Contreras C. Nutrition Day Colombia 2012. Bogotá, D.C.; 2012.

39. Hiesmayr JM, Schindler K, Kosak S. Nutrition Day in Colombia [Internet]. Vienna, AT; 2013 [Citado el 22 de mayo de 2016]. Disponible en: http://www.nutritionday.org/cms/upload/pdf/6_about_nutritionDay/6.4.national_reports_2013/ ND13_country_CO_EN.pdf

40. Keusch GT. The History of Nutrition: Malnutrition, Infection and Immunity. J Nutr. 2003;133:336-340S.

41. Giraldo Giraldo AN, Múnera García NE, Marrugo Espitaleta V, Piñeres LM. Prevalencia de malnutrición y evaluación de la prescripción dietética en pacientes adultos hospitalizados en una institución pública de alta complejidad. Perspect en Nutr Humana. 2007;9(1):37-47.

42. Lewis SJ, Egger M, Sylvester PA, Thomas S. Early enteral feeding versus "nil by mouth" after gastrointestinal surgery: systematic review and meta-analysis of controlled trials. BMJ. 2001;323(7316):773-6.

43. Cuervo Pinto JH. Ayuno preoperatorio: tiempos de cambio. Rev Col Anest. 1996;24(2):167-70.

44. Alvarez Plata L, Reyes Patino $\mathrm{RD}$. Ayuno preoperatorio en ninos sanos de 2, 4 y 6 horas. Rev Col Anest. 2009;37:63-70.
45. Manchikanti L, Malla Y, Wargo BW, Fellows B. Preoperative fasting before interventional techniques: is it necessary or evidence-based? Pain Physician. 2011 Jan;14(5):459-67.

46. Pimenta GP, de Aguilar-Nascimento JE. Prolonged preoperative fasting in elective surgical patients: why should we reduce it? Nutr Clin Pract. 2014;29(1):22-8.

47. Pexe-Machado PA, de Oliveira BD, Dock-Nascimento DB, de Aguilar-Nascimento JE. Shrinking preoperative fast time with maltodextrin and protein hydrolysate in gastrointestinal resections due to cancer. Nutrition. 2013;29(78):1054-9.

48. Koseoglu Z, Ozdogan M, Kuvvetli A, Kosenli O, Oruc C, Onel S, et al. Increased nutritional risk in major trauma: correlation with complications and prolonged length of stay. Turkish J Trauma Emerg Surg. 2011;17(6):521-4.

49. Meij BS Van Der, Schueren MAEVB Van Der, Langius JAE, Brouwer IA, Leeuwen PAM Van. n-3 PUFAs in cancer, surgery , and critical care: a systematic review on clinical effects, incorporation, and washout of oral or enteral compared with parenteral supplementation. Am J Clin Nutr. 2011;94:1248-65.

50. Mercadal Orfila G, Llop Talaverón JM. Effectiveness of perioperative glutamine in parenteral nutrition in patients at risk of moderate to severe malnutrition. Nutr Hosp. 2011;26(6):1305-12.

51. Cahill NE, Dhaliwal R, Day AG, Jiang $X$, Heyland DK. Nutrition therapy in the critical care setting: what is "best achievable" practice? An international multicenter observational study. Crit Care Med. 2010 Feb;38(2):395-401.

52. Pérez Gutiérrez N, Echeverry L, Mejía Sarmiento MA, López Acosta OA, Vega $S M$, Vélez Escobar CE, et al. Nutrición en el paciente con pancreatitis aguda grave: 
guía de manejo clínico. Rev Colomb Cir. 2010;25:104-20.

53. Pérez Gutiérrez N, Echeverry L, Forero A, Vega S, Villanueva O, Cruz JL, et al. Nutrición en pacientes críticos de la Orinoquia colombiana. Acta Colomb Cuid Intens. 2009;9(3):206-13.

54. Rugeles SJ. Nutrición y metabolismo en Cirugía: conferencia Rafael Casas Morales 2009. Rev Colomb Cir. 2009;24:223-8.

55. Ortiz Saavedra P, Manrique Hurtado $\mathrm{H}$, Solís Villanueva J, Candiotti Herrera M, Ige Afuso M, Torres Ruiz C. Prevalencia de desnutrición en los servicios de hospitalización de medicina. Rev Soc Peru Med Interna. 2007;20(1):16-20.

56. Kudsk KA, Croce MA, Fabian TC, Minard G, Tolley EA, Poret A, et al. Enteral versus parenteral feeding: Effects on septic morbidity after blunt and penetrating abdominal trauma. Ann Surg. 1992;215(5):503-11.

57. Dunn EL, Moore EE, Bohus RW. Immediate postoperative feeding following massive abdominal trauma: the catheter jejunostomy. JPEN. 1980;4(4):393-5.

58. Moore FA, Feliciano D V, Andrassy RJ, McArdle AH, Booth FVM, Morgenstein-Wagner TB, et al. Early enteral feeding, compared with parenteral, reduces postoperative septic complications: the results of a Meta-analysis. Ann Surg. 1992;216(2):172-83.

59. Moore FA, Moore EE, Jones T, McCroskey BL, Peterson VM. TEN versus TPN following major abdominal trauma: reduced septic morbidity. J Trauma. 1989;29(7):916-22.

60. Correia MITD, Waitzberg DL. The impact of malnutrition on morbidity, mortality, length of hospital stay and costs evaluated through a multivariate model analysis. Clin Nutr. 2003;22(3):235-9.
61. Hiesmayr M, Schindler K, Pernicka E, Schuh C, Schoeniger-Hekele A, Bauer $P$, et al. Decreased food intake is a risk factor for mortality in hospitalised patients: The NutritionDay survey 2006. Clin Nutr. 2009;28:484-91.

62. White J V, Guenter P, Jensen G, Malone A, Schofield M. Consensus statement of the Academy of Nutrition and Dietetics/American Society for Parenteral and Enteral Nutrition: characteristics recommended for the identification and documentation of adult malnutrition (undernutrition). J Acad Nutr Diet. Elsevier; 2012 May;112(5):730-8.

63. González González JL. Evaluación del estado nutricional de un grupo de adultos mayores pertenecientes al plan Nueva Sonrisa dispensario Santa Francisca Romana. Pontificia Universidad Javeriana; 2011. 22 páginas.

64. De Ulíbarri Pérez JI, González-Madrõo Giménez a., González Pérez P, Fernández $G$, Rodríguez Salvanés $F$, Mancha Álvarez-Estrada a., et al. Nuevo procedimiento para la detección precoz y control de la desnutrición hospitalaria. Nutr Hosp. 2002;17(4):179-88.

65. Pérez J, César M, Benavent E, Álvarez-Estrada a. Detección precoz y control de la desnutrición hospitalaria. Nutr Hosp. 2002;17(3):139-46.

66. Botina N DC, Ayala C VA, Paz Z IC, Limas C LA, Mafla AC. Estado nutricional y riesgo de malnutrición en pacientes hospitalizados del Hospital Universitario Departamental de Nariño. Rev Univ Ind Santander. 2013;45(3):5-17.

67. Ruiz JP, García O, Medina C. Malnutrición en pacientes con patología quirúrgica en cirugía general. Reper Med Cir. 2010;19(2):155-60.

68. Savino P. Desnutrición hospitalaria: grupos de soporte metabólico y nutricional. Primera parte. Rev Colomb Cir. 
INVESTIGACIONES ANDINA No. 33 Vol. 18

69. Bossio Bermúdez JE, Matson-CarbaIlo G. Valoración bioquímica preoperatoria y complicaciones de los pacientes de cirugía de cadera. Hospital Universitario del Caribe. Cartagena. Colombia. Rev Ciencias Biomédicas. 2011;2(2):256-61.

70. Tappenden K a., Quatrara B, Parkhurst ML, Malone AM, Fanjiang G, Zie- gler TR. Critical Role of Nutrition in Improving Quality of Care: An Interdisciplinary Call to Action to Address Adult Hospital Malnutrition. JPEN J Parenter Enter Nutr. 2013;37(4):482-99.

71. Cárdenas Zuluaga D. El papel del médico en la nutrición. Rev Salud Bosque. 2011;1(2):55-62. 